PROCEEDINGS OF THE

AMERICAN MATHEMATICAL SOCIETY

Volume 131, Number 10, Pages 3101-3107

S 0002-9939(03)06852-7

Article electronically published on January 28, 2003

\title{
QUASICONVEXITY AND UNIQUENESS OF STATIONARY POINTS IN THE MULTI-DIMENSIONAL CALCULUS OF VARIATIONS
}

\author{
ALI TAHERI \\ (Communicated by Bennett Chow)
}

\begin{abstract}
Let $\Omega \subset \mathbb{R}^{n}$ be a bounded starshaped domain. In this note we consider critical points $\bar{u} \in \bar{\xi} y+W_{0}^{1, p}\left(\Omega ; \mathbb{R}^{m}\right)$ of the functional

$$
\mathcal{F}(u, \Omega):=\int_{\Omega} f(\nabla u(y)) d y,
$$

where $f: \mathbb{R}^{m \times n} \rightarrow \mathbb{R}$ of class $\mathrm{C}^{1}$ satisfies the natural growth

$$
|f(\xi)| \leq c\left(1+|\xi|^{p}\right)
$$

for some $1 \leq p<\infty$ and $c>0$, is suitably rank-one convex and in addition is strictly quasiconvex at $\bar{\xi} \in \mathbb{R}^{m \times n}$. We establish uniqueness results under the extra assumption that $\mathcal{F}$ is stationary at $\bar{u}$ with respect to variations of the domain. These statements should be compared to the uniqueness result of Knops \& Stuart (1984) in the smooth case and recent counterexamples to regularity produced by Müller \& Šverák (2003).
\end{abstract}

\section{INTRODUCTION}

Let $\Omega \subset \mathbb{R}^{n}$ be a bounded starshaped domain and to avoid unnecessary technicalities assume that it has a $\mathrm{C}^{1}$ boundary. In this note we consider integral functionals of the form

$$
\mathcal{F}(u, \Omega):=\int_{\Omega} f(\nabla u(y)) d y,
$$

where $f: \mathbb{R}^{m \times n} \rightarrow \mathbb{R}$ is of class $\mathrm{C}^{1}$ and its gradient satisfies the growth condition

$$
|D f(\xi)| \leq c\left(1+|\xi|^{p-1}\right)
$$

for some $c>0$ and $1 \leq p<\infty$. We address the question of uniqueness for critical points of $\mathcal{F}$ subject to linear boundary conditions. We refer to a map $\bar{u} \in W^{1, p}\left(\Omega ; \mathbb{R}^{m}\right)$ as a critical point of the functional (1.1) if and only if the first variation of $\mathcal{F}$ subject to variations of $\bar{u}$ in the target space $\mathbb{R}^{m}$ is zero. This means that

$$
\left.\frac{d}{d \varepsilon} \mathcal{F}(\bar{u}+\varepsilon \varphi, \Omega)\right|_{\varepsilon=0}=\int_{\Omega} f_{\xi_{\alpha}^{i}}(\nabla \bar{u}(y)) \varphi_{, \alpha}^{i}(y) d y=0
$$

for all $\varphi \in \mathrm{C}_{0}^{\infty}\left(\Omega ; \mathbb{R}^{m}\right)$.

Received by the editors July 31, 2001 and, in revised form, April 24, 2002.

2000 Mathematics Subject Classification. Primary 49J10, 49J45.

(C)2003 American Mathematical Society 
Another condition of first order that we use in the sequel, is what is often refered to as stationarity. Here one introduces variations in the domain and demands the corresponding variation in the functional to vanish. More precisely one considers the one-parameter family of diffeomorphisms $\psi_{\varepsilon}: \mathbb{R}^{n} \rightarrow \mathbb{R}^{n}$ given by $\psi_{\varepsilon}(y)=y+\varepsilon \varphi(y)$ for arbitrary $\varphi \in \mathrm{C}_{0}^{\infty}\left(\mathbb{R}^{n} ; \mathbb{R}^{n}\right)$ with $\operatorname{supp} \varphi \subset \Omega$ and small enough $\varepsilon$. Setting $\bar{u}_{\varepsilon}(y)=\bar{u}\left(\psi_{\varepsilon}(y)\right)$ it should then follow that

$$
\left.\frac{d}{d \varepsilon} \mathcal{F}\left(\bar{u}_{\varepsilon}, \Omega\right)\right|_{\varepsilon=0}=\int_{\Omega}\left(f(\nabla \bar{u}(y)) \delta_{\alpha}^{\beta}-\bar{u}_{, \alpha}^{i}(y) f_{\xi_{\beta}^{i}}(\nabla \bar{u}(y))\right) \varphi_{, \beta}^{\alpha}(y) d y=0 .
$$

It can be easily checked that if $\bar{u}$ of class $\mathrm{C}^{1}$ is a weak local minimizer, or $\bar{u} \in W^{1, p}\left(\Omega ; \mathbb{R}^{m}\right)$ is a $W^{1, p}$ local minimizer of $\mathcal{F}$ (cf. [10] for terminology), then it satisfies the stationarity condition. In a similar way, if $f$ is of class $\mathrm{C}^{2}$ and $\bar{u}$ is a critical point also of class $\mathrm{C}^{2}$, then (1.4) holds. Under less regularity, equation (1.4) is genuinely different from (1.3).

We recall that a continuous function $f: \mathbb{R}^{m \times n} \rightarrow \mathbb{R}$ is said to be quasiconvex at $\xi \in \mathbb{R}^{m \times n}$ if and only if

$$
\mathcal{F}(\xi y, \Omega) \leq \mathcal{F}(u, \Omega)
$$

for every $u \in \xi y+W_{0}^{1, \infty}\left(\Omega, \mathbb{R}^{m}\right)$. Similarly $f$ is said to be rank-one convex at $\xi \in$ $\mathbb{R}^{m \times n}$ if and only if for every $\lambda \in \mathbb{R}^{m}$ and $\mu \in \mathbb{R}^{n}$ the function $\mathbb{R} \ni s \mapsto f(\xi+s \lambda \otimes \mu)$ is convex at $s=0$, that is, the inequality $f(\xi) \leq t f(\xi+\lambda \otimes \mu)+(1-t) f\left(\xi-\frac{t}{1-t} \lambda \otimes \mu\right)$ holds for all $0<t<1$. Finally $f$ is quasiconvex (rank-one convex) if and only if it is quasiconvex (rank-one convex) at every $\xi \in \mathbb{R}^{m \times n}$.

Note that if $f$ is rank-one convex, then it is locally Lipschitz. Moreover if it satisfies the growth condition

$$
|f(\xi)| \leq c\left(1+|\xi|^{p}\right)
$$

for some $c>0$ and $1 \leq p<\infty$, then (1.2) holds (for a possibly different constant $c>0$ and) for $\mathcal{L}^{m n}$-a.e. $\xi \in \mathbb{R}^{m \times n}$ (cf. e.g. [1]). We also note that (1.6) together with the Meyers-Serrin approximation theorem imply that (1.5) holds if we replace $W_{0}^{1, \infty}\left(\Omega ; \mathbb{R}^{m}\right)$ with $W_{0}^{1, p}\left(\Omega ; \mathbb{R}^{m}\right)$. If the latter inequality is strict for $u \neq \xi y$, then we say that $f$ is strictly quasiconvex at $\xi$.

The question of uniqueness for critical points of $\mathcal{F}$ subject to linear boundary conditions was first discussed by Knops \& Stuart [6]. There it is shown that under strict quasiconvexity of the integrand any $\mathrm{C}^{2}$ critical point of $\mathcal{F}$ in a starshaped domain and subject to the linear boundary condition $\bar{u}=\bar{\xi} y$ coincides with the linear map $\bar{\xi} y$ throughout $\Omega$. Their result relies on an integral representation for the energy of a critical point of class $\mathrm{C}^{2}$ in a bounded domain presented in [3] (cf. 6] Proposition 2.1). It turns out that the weak form of this identity is an immediate consequence of the stationarity condition (1.4).

It is important to observe that in such uniqueness statements some restriction needs to be imposed on the domain topology (cf. [13]). In the related setting of nonlinear elasticity, for $\Omega \subset \mathbb{R}^{2}$ an annulus, a heuristic argument of F. John [5] enables one to construct, even for linear boundary conditions such as $u=$ id, at least countably many smooth critical points for $\mathcal{F}$. The idea being that by defining appropriate homotopy classes in the corresponding mapping space (a representative of any such class would be a map that fixes the inner boundary and rotates the outer 
one by an integer multiple of $2 \pi$ ) one arrives at critical points of $\mathcal{F}$ by minimizing $\mathcal{F}$ on each such homotopy class. A recent article by Post \& Sivaloganathan (cf. [9]) studies this example in more detail. The effect of domain topology on the multiplicity of strong local minimizers of $\mathcal{F}$ on arbitrary Lipschitz domains $\Omega \subset \mathbb{R}^{2}$ is settled in [12].

In addition to the domain topology the regularity of the critical point in question also plays a significant role. Indeed Müller and Šverák [8 have constructed, for the case when $\Omega$ is the unit ball in $\mathbb{R}^{2}$, Lipschitz solutions (but no better) to (1.3) corresponding to a strictly quasiconvex integrand $f$ that vanish on the boundary, a sharp contrast to the uniqueness result in [6].

In this note we aim to study this gap and prove optimal uniqueness results for critical points of $\mathcal{F}$ when the stationarity condition (1.4) holds (in the sequel we refer to such maps as stationary points of $\mathcal{F}$ ). At this stage it is important to remind the reader that the role of (1.4) in diverse areas of geometric analysis has been observed by many authors (cf. [4] and the references therein for discussions in connection with, for example, various partial regularity theorems for harmonic maps).

An interesting question to pursue would be to see how far can one push the uniqueness result for linear boundary conditions, e.g. can one replace the assumption of $\Omega$ being starshaped by $\Omega$ being contractible? And can one classify domains with a nontrivial topology, ideally, in terms of their homology and homotopy groups? (Recall that for starshaped domains the well-known Poincaré lemma implies that all homology and homotopy groups in dimensions greater than zero are trivial.) We refer the interested reader to [12] and [13] for further results in this direction.

We end this introduction by observing how a simple dilatation argument establishes the required uniqueness result for $W^{1, p}$ local minimizers of $\mathcal{F}$ in starshaped domains (without loss of generality with respect to the origin) when $1 \leq p<\infty$. Indeed it follows from the growth condition (1.6) and the strict quasiconvexity of $f$ at $\bar{\xi}$ that the linear map $u_{0}=\bar{\xi} y$ is the absolute minimizer of $\mathcal{F}$ over $\bar{\xi} y+W_{0}^{1, p}\left(\Omega ; \mathbb{R}^{m}\right)$. Therefore if $u_{1} \neq u_{0}$ is a $W^{1, p}$ local minimizer of $\mathcal{F}$ in the above class, it must be that $\mathcal{F}\left(u_{0}, \Omega\right)<\mathcal{F}\left(u_{1}, \Omega\right)$. One can then consider the sequence $\left\{u_{\delta}\right\}$ for $\delta<1$ where

$$
u_{\delta}(y)= \begin{cases}\delta u_{1}\left(\frac{y}{\delta}\right) & \text { in } \Omega_{\delta}, \\ \bar{\xi} y & \text { in } \Omega \backslash \Omega_{\delta},\end{cases}
$$

and $\Omega_{\delta}=\delta \Omega$. It is easy to see that

$$
\mathcal{F}\left(u_{\delta}, \Omega\right)=\mathcal{F}\left(u_{1}, \Omega\right)+\left(1-\delta^{n}\right)\left(\mathcal{F}\left(u_{0}, \Omega\right)-\mathcal{F}\left(u_{1}, \Omega\right)\right),
$$

and so the contradiction is reached by noting that $u_{\delta} \rightarrow u_{1}$ in $W^{1, p}\left(\Omega ; \mathbb{R}^{m}\right)$ as $\delta \nearrow 1$.

\section{Proof of the main Result}

According to the starting assumption on the domain $\Omega$ and without loss of generality there exists a strictly positive function $d: \mathbb{S}^{n-1} \rightarrow \mathbb{R}$ of class $\mathrm{C}^{1}$ such that

$$
\partial \Omega=\left\{y \in \mathbb{R}^{n}:|y|=d(y /|y|)\right\}
$$


It is then clear that $\Omega=\{0\} \cup\left\{y \in \mathbb{R}^{n} \backslash\{0\}:|y|<d(\theta)\right\}$ where $\theta=y /|y|$ and therefore the unit outer normal to the boundary at a point $y \in \partial \Omega$ is simply

$$
\nu(y)=\alpha^{-1}(\theta)\left(\theta-(I-\theta \otimes \theta) \frac{\nabla d(\theta)}{d(\theta)}\right),
$$

with $\alpha(\theta)=d(\theta)^{-1}\left(d(\theta)^{2}+|\nabla d(\theta)|^{2}-(\theta \cdot \nabla d(\theta))^{2}\right)^{\frac{1}{2}}$.

We now claim that for every $f \in L^{1}(\Omega)$,

$$
\int_{\Omega} f(y) d y=\int_{0}^{1} \rho^{n-1} \int_{\partial \Omega} \frac{d(\theta)}{\alpha(\theta)} f(\rho y) d \mathcal{H}^{n-1}(y) d \rho .
$$

A straightforward proof of this assertion follows from the co-area formula (cf. Federer [2, Theorem 3.2.12, p. 249) with the particular choice of $f(x)=|x| / d(x /|x|)$ there and noting that $d$ and $\alpha$ are bounded away from zero. An alternative way of achieving this is to consider the map $F: B^{n} \rightarrow \Omega$ given by $F(x)=x d(x /|x|)$ when $x \neq 0$ and $F(0)=0$. Then for $x \neq 0$

$$
\nabla F(x)=d(\omega) I+\omega \otimes(I-\omega \otimes \omega) \nabla d(\omega),
$$

where $I$ denotes the identity matrix and $\omega=x /|x|$. Since $F$ is invertible and its Jacobian is $\operatorname{det} \nabla F(x)=d^{n}(\omega)$, we can immediately deduce that

$$
\begin{aligned}
\int_{\Omega} f(y) d y & =\int_{B^{n}} f(F(x)) d^{n}(\omega) d x \\
& =\int_{0}^{1} \rho^{n-1} \int_{\mathbb{S}^{n-1}} f(F(\rho \omega)) d^{n}(\omega) d \mathcal{H}^{n-1}(\omega) d \rho
\end{aligned}
$$

for every $f \in L^{1}(\Omega)$. With the particular choice of $f_{\varepsilon}=\chi_{\{y \in \Omega \text { : } \operatorname{dist}(y, \partial \Omega) \leq \varepsilon\}}$, this formula leads to the following identity:

$$
\mathcal{H}^{n-1}(\partial \Omega)=\int_{\mathbb{S}^{n-1}} \alpha(\omega) d^{n-1}(\omega) d \mathcal{H}^{n-1}(\omega) .
$$

Using a similar formula for relatively open subsets of $\partial \Omega$, arguing first for characteristic functions and then passing to the limit, we have for every $g \in L^{1}(\partial \Omega)$

$$
\int_{\partial \Omega} g(y) d \mathcal{H}^{n-1}(y)=\int_{\mathbb{S}^{n-1}} \alpha(\omega) g(\omega d(\omega)) d^{n-1}(\omega) d \mathcal{H}^{n-1}(\omega) .
$$

Setting $g(y)=\frac{d(\theta)}{\alpha(\theta)} f(\rho y)$ in the above identity and substituting in (2.2) gives the conclusion.

We continue by considering the homogeneous degree-one extension map $\bar{u}^{\text {hom }}$ : $\Omega \rightarrow \mathbb{R}^{m}$, associated with a given map $\bar{u} \in W^{1, p}\left(\partial \Omega ; \mathbb{R}^{m}\right)$. This is defined as $\bar{u}^{\text {hom }}(y)=(|y| / d(\theta)) \bar{u}(\theta d(\theta))$. It can be easily checked that

$$
\nabla \bar{u}^{h o m}(y)=\nabla \bar{u}(\theta d(\theta))+\left(\frac{\bar{u}(\theta d(\theta))}{d(\theta)}-\nabla \bar{u}(\theta d(\theta)) \theta\right) \otimes\left(\theta-(I-\theta \otimes \theta) \frac{\nabla d(\theta)}{d(\theta)}\right) .
$$

Assume now that $f: \mathbb{R}^{m \times n} \rightarrow \mathbb{R}$ is a given $\mathrm{C}^{1}$ function that is rank-one convex at points $\xi \in \mathbb{R}^{m \times n}$ with $\xi=\nabla \bar{u}(y)$ for $y \in \partial \Omega$. Then using the inequality $f(\xi+\lambda \otimes \mu) \geq f(\xi)+\langle D f(\xi), \lambda \otimes \mu\rangle$ that holds for all $\lambda \in \mathbb{R}^{m}$ and $\mu \in \mathbb{R}^{n}$ and 
(2.1) above we can write

$$
\begin{aligned}
& \int_{\Omega} n f\left(\nabla \bar{u}^{\text {hom }}(y)\right) d y \\
& =\int_{\partial \Omega} \frac{d(\theta)}{\alpha(\theta)} f\left(\nabla \bar{u}(y)+\left(\frac{\bar{u}(y)}{d(\theta)}-\nabla \bar{u}(y) \theta\right)\right. \\
& \left.\qquad\left(\theta-(I-\theta \otimes \theta) \frac{\nabla d(\theta)}{d(\theta)}\right)\right) d \mathcal{H}^{n-1}(y) \\
& \geq \int_{\partial \Omega} \frac{d(\theta)}{\alpha(\theta)} f(\nabla \bar{u}(y)) d \mathcal{H}^{n-1}(y) \\
& +\int_{\partial \Omega} \frac{1}{\alpha(\theta)}\left\langle D f(\nabla \bar{u}(y)), \bar{u}(y) \otimes\left(\theta-(I-\theta \otimes \theta) \frac{\nabla d(\theta)}{d(\theta)}\right)\right\rangle d \mathcal{H}^{n-1}(y) \\
& -\int_{\partial \Omega} \frac{d(\theta)}{\alpha(\theta)}\left\langle D f(\nabla \bar{u}(y)), \nabla \bar{u}(y) \theta \otimes\left(\theta-(I-\theta \otimes \theta) \frac{\nabla d(\theta)}{d(\theta)}\right)\right\rangle d \mathcal{H}^{n-1}(y) .
\end{aligned}
$$

We now proceed by taking the particular choice $\varphi(y)=r_{\varepsilon, t}(|y| / d(\theta)) y$ in (1.4) for $0<t \leq 1$ and $\varepsilon>0$ where

$$
r_{\varepsilon, t}(s)= \begin{cases}1 & \text { for } 0 \leq s \leq t-\varepsilon \\ 1-\frac{s-(t-\varepsilon)}{\varepsilon} & \text { for } t-\varepsilon \leq s \leq t, \\ 0 & \text { for } t \leq s \leq 1\end{cases}
$$

that is clearly allowed by approximation. One can then easily verify that

$$
\nabla \varphi(y)=r_{\varepsilon, t}\left(\frac{|y|}{d(\theta)}\right) I+\frac{1}{d(\theta)} r_{\varepsilon, t}^{\prime}\left(\frac{|y|}{d(\theta)}\right) y \otimes\left(\theta-(I-\theta \otimes \theta) \frac{\nabla d(\theta)}{d(\theta)}\right) .
$$

Substituting this into the equation, we have

$$
\begin{aligned}
& \int_{\Omega} n r_{\varepsilon, t}\left(\frac{|y|}{d(\theta)}\right) f(\nabla \bar{u}(y)) d y \\
& =\int_{\Omega} \frac{-1}{d(\theta)} r_{\varepsilon, t}^{\prime}\left(\frac{|y|}{d(\theta)}\right) y \cdot\left(\theta-(I-\theta \otimes \theta) \frac{\nabla d(\theta)}{d(\theta)}\right) f(\nabla \bar{u}(y)) d y \\
& +\int_{\Omega} r_{\varepsilon, t}\left(\frac{|y|}{d(\theta)}\right)\langle\nabla \bar{u}(y), D f(\nabla \bar{u}(y))\rangle d y \\
& +\int_{\Omega} \frac{1}{d(\theta)} r_{\varepsilon, t}^{\prime}\left(\frac{|y|}{d(\theta)}\right)\left\langle D f(\nabla \bar{u}(y)), \nabla \bar{u}(y) y \otimes\left(\theta-(I-\theta \otimes \theta) \frac{\nabla d(\theta)}{d(\theta)}\right)\right\rangle d y \\
& =\mathrm{I}+\mathrm{II}+\mathrm{III} .
\end{aligned}
$$

Using (2.1) and Lebesgue's differentiation theorem we have for $\mathcal{L}^{1}$-a.e. $t \in(0,1]$

$$
\begin{aligned}
\lim _{\varepsilon \searrow 0} \mathrm{I} & =\lim _{\varepsilon \searrow 0} \varepsilon^{-1} \int_{t-\varepsilon}^{t} \rho^{n} \int_{\partial \Omega} \frac{d(\theta)}{\alpha(\theta)} f(\nabla \bar{u}(\rho y)) d \mathcal{H}^{n-1}(y) d \rho \\
& =\int_{\partial \Omega_{t}} t \frac{d(\theta)}{\alpha(\theta)} f(\nabla \bar{u}(y)) d \mathcal{H}^{n-1}(y),
\end{aligned}
$$

where $\Omega_{t}=t \Omega$. Using a similar argument for III it can be shown that again for $\mathcal{L}^{1}$-a.e. $t \in(0,1]$ the limit as $\varepsilon \searrow 0$ exists and equals

$$
\begin{aligned}
& \lim _{\varepsilon \searrow 0} \text { III } \\
& =-\int_{\partial \Omega_{t}} t \frac{d(\theta)}{\alpha(\theta)}\left\langle D f(\nabla \bar{u}(y)), \nabla \bar{u}(y) \theta \otimes\left(\theta-(I-\theta \otimes \theta) \frac{\nabla d(\theta)}{d(\theta)}\right)\right\rangle d \mathcal{H}^{n-1}(y) .
\end{aligned}
$$


Recalling that $r_{\varepsilon, t} \rightarrow \chi_{\Omega_{t}}$ in $L^{1}(\Omega)$ as $\varepsilon \searrow 0$, using (1.2) and Lebesgue's theorem on dominated convergence, we can pass to the limit in II. Applying (1.3) and ignoring a further subset of $(0,1]$ with zero $\mathcal{L}^{1}$-measure we can re-write this limit as

$$
\int_{\Omega_{t}}\langle\nabla \bar{u}(y), D f(\nabla \bar{u}(y))\rangle d y=\int_{\partial \Omega_{t}}\langle\bar{u}(y) \otimes \nu(y), D f(\nabla \bar{u}(y))\rangle d \mathcal{H}^{n-1}(y),
$$

where as before $\nu$ is the unit outward normal to the boundary $\partial \Omega_{t}$. Based on the above argument, we can deduce from (2.4) after passing to the limit $\varepsilon \searrow 0$, that for $\mathcal{L}^{1}$-a.e. $t \in(0,1]$

$$
\begin{aligned}
& \int_{\Omega_{t}} n f(\nabla \bar{u}(y)) d y \\
& =\int_{\partial \Omega_{t}} t \frac{d(\theta)}{\alpha(\theta)} f(\nabla \bar{u}(y)) d \mathcal{H}^{n-1}(y)+\int_{\partial \Omega_{t}}\langle\bar{u}(y) \otimes \nu(y), D f(\nabla \bar{u}(y))\rangle d \mathcal{H}^{n-1}(y) \\
& -\int_{\partial \Omega_{t}} t \frac{d(\theta)}{\alpha(\theta)}\left\langle D f(\nabla \bar{u}(y)), \nabla \bar{u}(y) \theta \otimes\left(\theta-(I-\theta \otimes \theta) \frac{\nabla d(\theta)}{d(\theta)}\right)\right\rangle d \mathcal{H}^{n-1}(y) .
\end{aligned}
$$

Comparing this to the right-hand side of (2.3) after replacing $d(\theta)$ with $t d(\theta)$, leads us to the following statement.

Proposition 2.1. Assume that $\Omega \subset \mathbb{R}^{n}$ is as above, $f$ of class $\mathrm{C}^{1}$ satisfies (1.2) and that $\bar{u} \in W^{1, p}\left(\Omega ; \mathbb{R}^{m}\right)$ is a stationary point of $\mathcal{F}(\cdot, \Omega)$. Then there exists $E \subset(0,1]$ with $\mathcal{L}^{1}(E)=0$ such that if $t \in(0,1] \backslash E$ and $f$ is rank-one convex at $\nabla \bar{u}(y)$ for $\mathcal{H}^{n-1}$-a.e. $y \in \partial \Omega_{t}$, then

$$
\mathcal{F}\left(\bar{u}, \Omega_{t}\right) \leq \mathcal{F}\left(\bar{u}_{t}^{\text {hom }}, \Omega_{t}\right)
$$

where $\bar{u}_{t}^{\text {hom }}$ is the homogeneous degree-one extension map corresponding to $\left.\bar{u}\right|_{\partial \Omega_{t}}$.

As mentioned earlier in the Introduction if $f$ is rank-one convex and satisfies (1.6), then (1.2) holds. Thus the conclusion of the above proposition holds in this particular case. It is now clear that if the restriction $\left.\bar{u}\right|_{\partial \Omega_{t}}$ is linear, then we have the following uniqueness result.

Theorem 2.1. Let $\Omega, f$ and $\bar{u}$ satisfy the hypotheses of the previous proposition and additionally that for $t \in(0,1] \backslash E$ the restriction $\left.\bar{u}\right|_{\partial \Omega_{t}}=\bar{\xi} y$ with $\bar{\xi} \in \mathbb{R}^{m \times n}$. Then the strict quasiconvexity of $f$ at $\bar{\xi}$ implies $\bar{u}(y)=\bar{\xi} y$ in $\Omega_{t}$.

Proof. We have $\bar{u}_{t}^{\text {hom }}(y)=\bar{\xi} y$. The conclusion now follows from the previous proposition.

Corollary 2.1. Assume that $\Omega$ and $f$ are as in Proposition 2.1 and that $\bar{u}$ of class $\mathrm{C}^{1}$ is a weak local minimizer of $\mathcal{F}$ with $\left.\bar{u}\right|_{\partial \Omega}=\bar{\xi}$ y and $f$ being rank-one convex at $\nabla \bar{u}(y)$ for $\mathcal{H}^{n-1}$-a.e. $y \in \partial \Omega$. Then the quasiconvexity of $f$ at $\bar{\xi}$ implies $\bar{u}$ to be an absolute minimizer of $\mathcal{F}$ in $\bar{\xi} y+W_{0}^{1, p}\left(\Omega ; \mathbb{R}^{m}\right)$. Moreover if $f$ is strictly quasiconvex at $\bar{\xi}$, then $\bar{u}=\bar{\xi} y$.

Remark 2.1. The conclusion of the above corollary does not hold if $\bar{u}$ is a weak local minimizer that is only Lipschitz (cf. [7] for a particular class of examples). This shows that the statement of the corollary is optimal.

Remark 2.2. It is possible to show that in the case of $W^{1, p}$ local minimizers $(1 \leq$ $p<\infty)$ there exists $1<\tau$ sufficiently close to 1 such that the map

$$
v= \begin{cases}\bar{u} & \text { in } \Omega, \\ \bar{\xi} y & \text { in } \Omega_{\tau} \backslash \Omega\end{cases}
$$


is a $W^{1, p}$ local minimizer of $\mathcal{F}\left(\cdot, \Omega_{\tau}\right)$. Indeed if this were not the case, there would exist a monotonically decreasing sequence $\tau_{j} \searrow 1$ and a sequence $v_{j} \in$ $\bar{\xi} y+W_{0}^{1, p}\left(\Omega_{\tau_{j}} ; \mathbb{R}^{m}\right)$ with $v_{j} \rightarrow v$ in $W^{1, p}\left(\Omega_{\tau_{1}} ; \mathbb{R}^{m}\right)$ such that $\mathcal{F}\left(v_{j}, \Omega_{\tau_{j}}\right)<\mathcal{F}\left(v, \Omega_{\tau_{j}}\right)$. Setting $u_{j}(y)=\tau_{j}^{-1} v_{j}\left(\tau_{j} y\right)$ for $y \in \Omega$, it follows that

$$
\mathcal{F}\left(u_{j}, \Omega\right)+\left(\tau_{j}^{n}-1\right) \mathcal{F}\left(u_{j}, \Omega\right)=\mathcal{F}\left(v_{j}, \Omega_{\tau_{j}}\right)<\mathcal{F}(\bar{u}, \Omega)+\left(\tau_{j}^{n}-1\right) \mathcal{F}(\bar{\xi} y, \Omega) .
$$

Thus using the quasiconvexity of $f$ at $\bar{\xi}$ and recalling that $u_{j} \in \bar{\xi} y+W_{0}^{1, p}\left(\Omega ; \mathbb{R}^{m}\right)$ the contradiction is reached once we show that $u_{j} \rightarrow \bar{u}$ in $W^{1, p}\left(\Omega ; \mathbb{R}^{m}\right)$. But

$$
\begin{aligned}
\tau_{j}^{n} & \int_{\Omega}\left|\nabla\left(u_{j}(y)-\bar{u}(y)\right)\right|^{p} d y \\
& =\int_{\Omega} \tau_{j}^{n}\left|\nabla v_{j}\left(\tau_{j} y\right)-\nabla \bar{u}(y)\right|^{p} d y=\int_{\Omega_{\tau_{j}}}\left|\nabla v_{j}(y)-\nabla \bar{u}\left(\frac{y}{\tau_{j}}\right)\right|^{p} d y \\
& \leq \int_{\Omega_{\tau_{j}}}\left|\nabla\left(v_{j}(y)-v(y)\right)\right|^{p} d y+\int_{\Omega_{\tau_{j}}}\left|\nabla v(y)-\nabla \bar{u}\left(\frac{y}{\tau_{j}}\right)\right|^{p} d y \\
& =\int_{\Omega_{\tau_{j}}}\left|\nabla\left(v_{j}(y)-v(y)\right)\right|^{p} d y+\int_{\Omega_{\tau_{j}}}\left|\nabla v(y)-\nabla v\left(\frac{y}{\tau_{j}}\right)\right|^{p} d y .
\end{aligned}
$$

The first term goes to zero by the definition of $v_{j}$ and the second term goes to zero by the continuity of dilatations. The claim is thus justified.

\section{REFERENCES}

[1] B. Dacorogna, Direct methods in the calculus of variations, Applied Mathematical Sciences 78, Springer-Verlag, 1988. MR 90e:49001

[2] H. Federer, Geometric measure theory, Graduate Texts in Mathematics 153, SpringerVerlag, 1969.

[3] A.E. Green, On some general formulae in finite elastostatics, Arch. Rational Mech. Anal., Vol. 50, 1973, pp. 73-80. MR 55:13931

[4] R.M. Hardt, Singularities of harmonic maps, Bull. Amer. Math. Soc., Vol. 34, No. 1, 1997, pp. 15-34. MR 98b:58046

[5] F. John, Uniqueness of nonlinear equilibrium for prescribed boundary displacement and sufficiently small strains, Comm. Pure Appl. Math., Vol. 25, 1972, pp. 617-634. MR 47:3857

[6] R.J. Knops, C.A. Stuart, Quasiconvexity and uniqueness of equilibrium solutions in nonlinear elasticity, Arch. Rational Mech. Anal., Vol 86, No. 3, 1984, pp. 233-249. MR 85j:73012

[7] J. Kristensen, A. Taheri, Partial regularity of strong local minimizers in the multidimensional calculus of variations, Max-Planck-Institute MIS (Leipzig) Preprint-Nr. 59, 2001.

[8] S. Müller, V. Šverák, Convex integration for Lipschitz mappings and counterexamples to regularity, To appear in Ann. of Math., 2003.

[9] K. Post, J. Sivaloganathan, On homotopy conditions and the existence of multiple equilibria in finite elasticity, Proc. Roy. Soc. Edin. A, Vol. 127, 1997, pp. 595-614. MR 98h:73030a

[10] A. Taheri, Sufficiency theorems for local minimizers of the multiple integrals of the calculus of variations, Proc. Roy. Soc. Edin. A, Vol. 131, 2001, pp. 155-184. MR 2002e:49036

[11] A. Taheri, On critical points of functionals with polyconvex integrands, J. Convex Anal., Vol. 9, 2002, pp. 55-72.

[12] A. Taheri, On Artin's braid group and polyconvexity in the calculus of variations, To appear in J. Lond. Math. Soc., 2002.

[13] A. Taheri, Local minimizers and quasiconvexity - the impact of Topology, Max-PlanckInstitute MIS (Leipzig) Preprint-Nr. 27, 2002.

Max-Planck-Institute for Mathematics in the Sciences, Inselstr. 22-26, D-04103 LeIPZig, Germany

E-mail address: taheri@mis.mpg.de 